\title{
Partitioning of a Hybrid Lipid in Domains of Saturated and Unsaturated Lipids in a
}

\section{Model Cellular Membrane}

\author{
Prashant Hitaishi, Priya Mandal and Sajal K. Ghosh* \\ Department of Physics, School of Natural Sciences, Shiv Nadar University, \\ Gautam Buddha Nagar, Uttar Pradesh 201314, India
}

1. X-ray reflectivity (XRR) from lipid multilayers: In the ternary system, the hybrid lipid POPC is added in the DPPC/DOPC mixture to get 0,20 and $40 \mathrm{~mol} \%$ of the lipid. XRR data at $85 \% \mathrm{RH}$ is added in the main text of the paper which is used further to calculate the dspacing and correlation length. The profiles of the mixed lipid systems obtained at $95 \%$ and 98\% relative humidity $(\mathrm{RH})$ are shown in figure $\mathrm{S} 1$ (left) and $\mathrm{S} 1$ (right) respectively. The two different sets of lamellar peaks signify the presence of two coexisting phases in the samples.
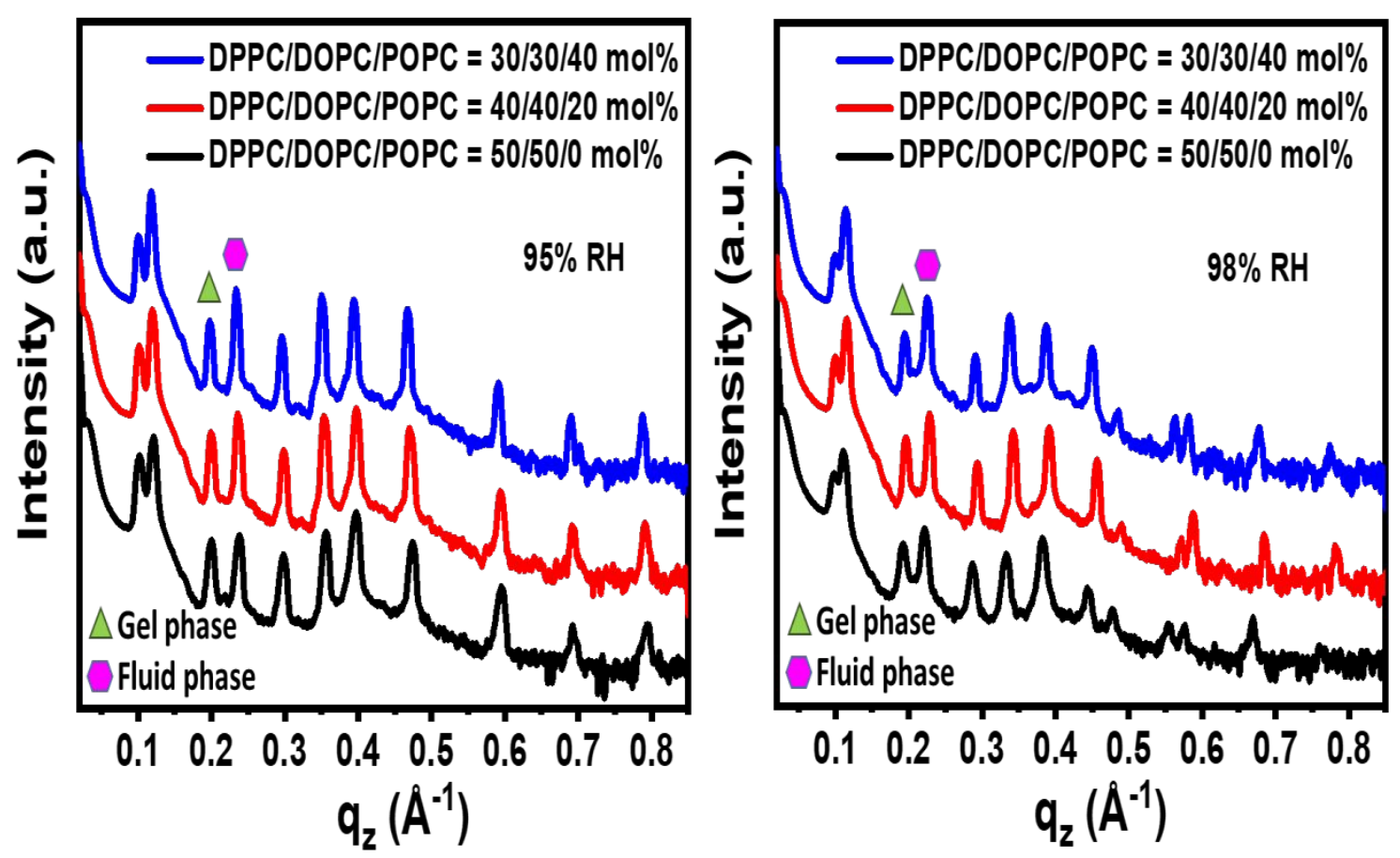

Figure S1: X-ray reflectivity (XRR) data of DPPC/DOPC/POPC system in presence of 0 , 20 and $40 \mathrm{~mol} \%$ of POPC at $95 \%$ (left) and $98 \%$ (right) relative humidity (RH). 
2. Phase factors of mixed lipid system: Phase factors for DPPC/DOPC/POPC mixed lipid system at 0,20, $40 \mathrm{~mol} \%$ concentration of POPC corresponding to gel and fluid phase are shown in figure S2. While the phase factors for gel phase are shown in figure S2 (a), (b) and (c) respectively, the same are shown for fluid phase in figure S2 (d), (e) and (f) respectively. The values are taken to be +1 or -1 considering the mirror symmetry in the lipid bilayer. As observed, these values depend on the lipid composition of a sample.
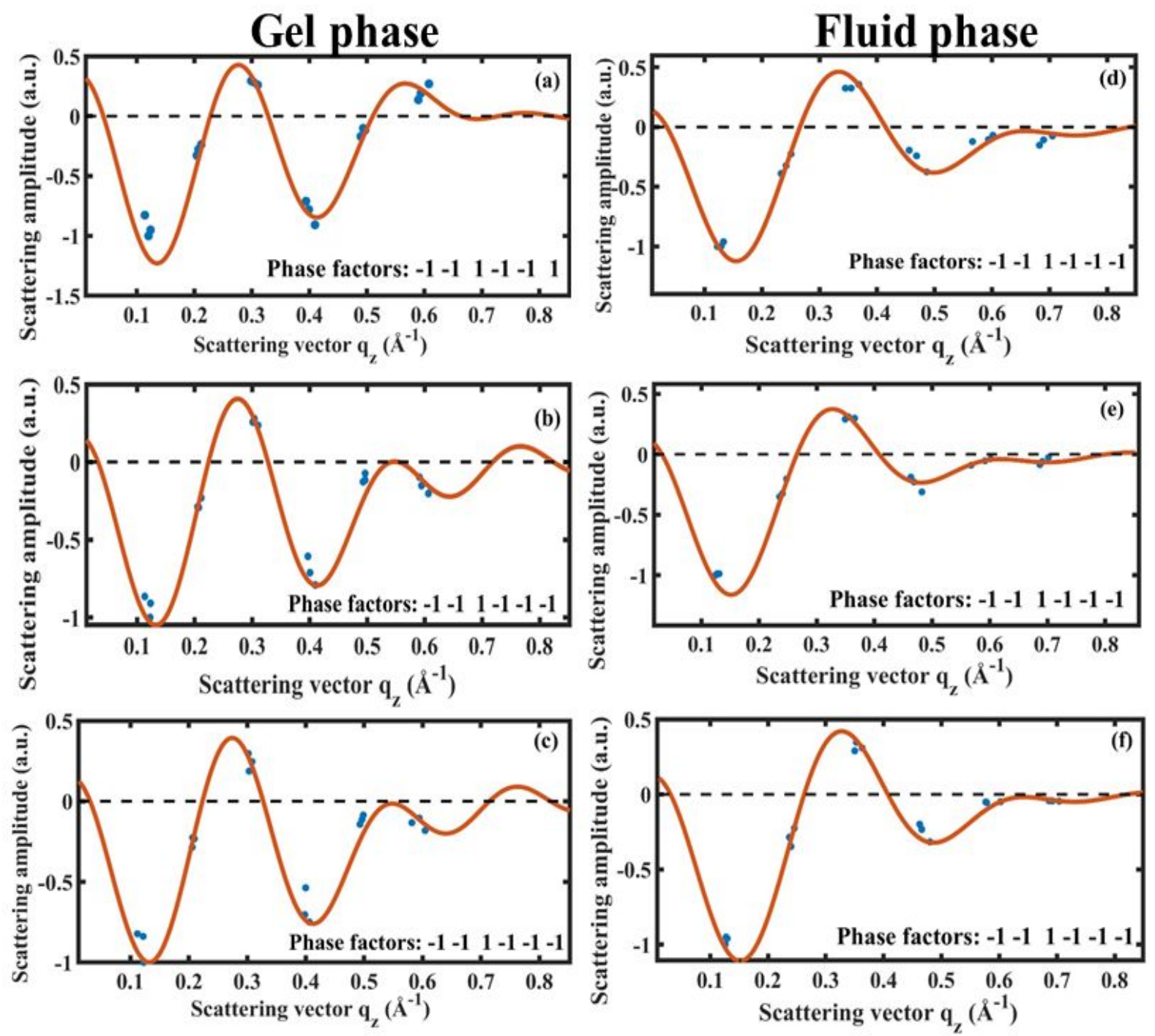

Figure S2: Determination of phase factor by applying swelling method for DPPC/DOPC/POPC system gel-phase in the presence of (a) 0, (b) 20, (c) 40 mol\% POPC and fluid-phase in the presence of (d) 0 , (e) $20,(f) 40 \mathrm{~mol} \%$ POPC. 